\title{
UN CAPÍTULO POCO CONOCIDO SOBRE LA HISTORIA DE LA PATATA EN LA REGIÓN PARISINA DURANTE EL SIGLO XIX ${ }^{1}$
}

\author{
LAURENT HERMENT \\ Centre de Recherches Historique CNRS-EHESS, UMR 8558
}

\begin{abstract}
RESUMEN. Las patatas constituyeron una de las plantas americanas que permitiern a los países europeos enfrentar su crecimiento demográfico entre finales del Antiguo Régimen y el remate del siglo XIX. Al contrario de lo sucedido en los países de la Europa septentrional, Galicia y el norte de Portugal, y con muy pocas excepciones regionales, en Francia, la patata no jugó un papel relevante en la alimentación de la población. Pese a ello, en algunas regiones, como por ejemplo en la cuenca de Paris, el Bassin Parisien, fue un cultivo muy importante. En esta zona, su rendimiento se multiplicó por diez entre finales del Primer Imperio y la década de 1860. A pesar de ello, no formaba parte de la dieta de la población rural, sino que era, más bien, un cultivo industrial utilizado por la industria del fécula para producir, entre otras cosas, glucosa y alcohol. Más allá del caso de la mencionada cuenca de Paris, la hipótesis de este trabajo es que la patata solo habría llegado a integrarse en la dieta de la población francesa si el precio del combustible (madera, carbón o turba) y el de la grasa animal o vegetal hubiesen sido bajos.

Palabras clave: patata, Bassin Parisien, fábrica de fécula, dieta.
\end{abstract}

Abstract. Potatoes constituted one of the most important American plants to allow European countries to face the demographic growth during the end of the Early Modern History and the

Recibido: 30-5-2018 - Aceptado: 28-6-2018 • laurent.herment@ehess.fr

Quiero agradecer a Juan Carmona la lectura crítica de la primera versión de este trabajo. Asimismo agradecer también los comentarios de los participantes en el coloquio internacional: Cultivos americanos, transformaciones agropecuarias e implicaciones del desarrollo de su comercio en Europa y América, siglos XVI-XX, celebrado en Santiago de Compostela el 9-10 de octubre de 2017 bajo los auspicios del GIHM-1921 y el GDRI-AAA (CNRS). Finalmente, quiero agradecer asimismo a los dos evaluadores anónimos sus consideraciones, las cuales me permitieron mejorar la última versión de este texto. 
nineteenth century. But, contrary to what occurred in North-European countries, Galicia and north of Portugal, with few regional exceptions, potato did not play a major role in the food intake of French population. Nevertheless potatoes had constituted in some regions, in Paris Bassin for example, a very important crop. In this region, yield of potato was multiplied tenfold between the end of the First Empire and the 1860s. Despite this increasing production, I show that potato was not an element of the diet of the Paris Bassin's rural population but was an industrial crop used by starch industry (industrie de la fécule in French) to produce, among other things, glucose and alcohol. Beyond the case of the Paris Bassin, I hypothesis that potato can become an element of the diet of the populations only if the price of fuel (wood, coal, charcoal or peat) and the price animal fat or vegetable fat were low.

Keywords: Potato, Paris Basin, Starch factory (potato), food intake.

EN LOS SIGLOS XVIII y XIX, las plantas de origen extra-europeo desempeñaron un papel fundamental en la evolución del régimen alimentario de las poblaciones de determinados países del Viejo Mundo. Así, Sydney Mintz demostró que el azúcar y el té jugaron un importante papel en la transformación de la dieta inglesa (Mintz, 1986). En otros lugares del continente, sabemos que el café consiguió sustituir sin grandes problemas al té (Bennezon, 2012), mientras que en muchas regiones europeas, el maíz fue el elemento que les permitió hacer frente a sus respectivos crecimientos poblacionales ${ }^{2}$. Sea como fuere, de entre todas las plantas importadas de América ninguna parece haber tenido tanta importancia como la patata, al menos hasta mediados del siglo XIX, cuando el tubérculo fue golpeado por la conocida como «peste de la patata» (Paping, Vanhaute y O'Grada, 2007). No obstante, tal y como señaló Nadine Vivier (2007), un desastre que afectó muy poco a Francia, todo lo contrario de lo que ocurrio en Irlanda y, en menor medida, en Flandes y los Países Bajos (Bergman, 1967).

A pesar de que en el siglo XIX la patata ocupó un lugar destacado en los sistemas agrarios y alimenticios de ciertas regiones de Francia, la planta no fue universalmente apreciada en el país y, en no pocas ocasiones, su cultivo fue marginal. A principios del siglo XIX, la encontramos sobre todo en las áreas de montaña - como los Vosgos, el Macizo Central o Saboya-, y en menor medida, en el oeste - caso de Bretaña y la parte más pobre de Normandía-, también en la llanura de Alsacia y, algo más tarde, en el norte (Dubuc, 1953; Boehler, 1995, pp. 755-769). En todas estas

En Francia, el maíz se implantó bastante pronto en el suroeste, en el Franco-Condado (Frèche, 1974, pp. 213-230; Meyzie, 2007, pp. 203 et ss.; Desplat, 1992, p. 1321; Boehler, 1995, pp. 752-755). En cuanto a las demás regiones europeas, remitimos al lector a los artículos que forman parte de este monográfico. 
áreas, ocupaba un lugar más o menos importante en la dieta de la población (Vandenbroeke, 1990). Por el contrario, en el Bassin Parisien la situación fue diferente. Al respecto, no deja de sorprender la falta de interés por el tubérculo en una región cuyo suelo se adaptaba bastante bien a su cultivo, además de ser una de las zonas más pobladas de Europa. Pero, como mostraron Steven Kaplan y Nicolas Bourguinat, al pueblo de París y a sus consejeros municipales les preocupaba más el abasto de trigo y harina a la ciudad que ocuparse en promocionar el consumo de patatas (Kaplan, 1984; Bourguinat, 2002; asimismo, ver a Herment y Ronsijn, 2015 y Ronsijn y Herment, 2016).

No obstante, a pesar de esta falta de éxito, a veces los científicos de la época alababan el tubérculo, destacando en esto el famoso Antoine-Augustin Parmentier (Spary 2016, pp. 55-87; Mc Neil, 1999). En todo caso, conviene reconocer que en el Bassin Parisien se lo consideraba un cultivo que era susceptible tanto de responder a situaciones de crisis y emergencia alimentaria cuando el trigo escaseaba, como de ser uno de los últimos recursos ante una carestía inminente. Si los tiempos eran de normalidad poca gente se preocupaba por la patata, lo que sin duda explica el desinterés que los historiadores de los sistemas agrarios mostraron en su día por el estudio de lo sucedido al respecto en la cuenca de París. Por nuestra parte, sin embargo, sí que hemos podido aportar alguna que otra información acerca de su cultivo en la región durante la primera mitad del siglo XIX, aunque debemos reconocer que, como la mayor parte de esos historiadores, en su momento no consideramos oportuno detenernos a estudiar con precisión qué era o que pasaba con él (Herment, 2012, pp. 199-201). Procedimos así como Jean-Marc Moriceau y Gilles Postel-Vinay, quienes en su estudio sobre los grandes agricultores de la región parisina ni lo nombran (Moriceau y Postel-Vinay, 1992), muestra de la escasa relevancia que tuvo, no sólo en las fuentes históricas que remiten a la historia rural de la zona, sino también, y sin duda, en la vida cotidiana y el imaginario alimentario de sus habitantes.

Los especialistas en historia cultural y los historiadores de la alimentación fueron los primeros que se interesaron por los usos de la patata (Faivre, 2011; Barrière, 2011; Tebben, 2011). En Francia, fueron las «french fries» y las distintas recetas que la tomaban como base lo que llamó la atención de la historiografía especializada ${ }^{3}$. Sin embargo, más allá de la patata está su cultivo, ya que antes de comerla hay que plantarla. Por esta razón, en este trabajo nos centraremos en el estudio de todo lo relativo a su cultivo en la región de París, en el Bassin Parisien.

A parte de las «french fries», existen numerosas recetas locales: gratin dauphinois, hachis parmentier, aligot, etc. 
Durante mucho tiempo, la historiografía rural partió de la hipótesis de que en dicha región, y en general todo el norte de Francia, la patata se cultivaba casi en exclusiva a pequeña escala: o bien lo hacían los horticultores de los suburbios de París con el objetivo de abastecer al mercado de la ciudad, o bien la cultivaban los jornaleros de sus alrededores en pequeñas parcelas de tierra como parte de una estrategia de supervivencia, o bien estaba presente en las huertas de las grandes granjas de la zona para satisfacer tanto el consumo humano como, y sobre todo, el de los animales. Unas presunciones que convendrá examinar en detalle, pues nunca han sido sometidas a la más mínima comprobación. De hecho, si nos fijamos en las estadísticas de producción del siglo XIX, advertimos que las superficies de terreno dedicadas a la patata en la zona eran lo suficientemente importantes como para que no podamos considerarla solo un cultivo hortícola o de jornaleros. Es más, en otras regiones de Francia fue cultivada in extenso, como por ejemplo ocurría en una parte de Lorena, al este del país, o en los departamentos del norte y de Pas-de-Calais, próximos a la frontera belga ¿Cómo explicar entonces que en estas zonas los grandes agricultores hayan consagrado decenas de miles de hectáreas, un cultivo que durante mucho tiempo la población se negó a comer? Si podemos demostrar que los habitantes de estas regiones no se alimentaban habitualmente de patatas, convendría interesarse pues por el destino de su producción. Lo sucedido en el Bassin Parisien, nos ayudará a averiguar qué era lo que pasaba al respecto.

La historia de los sistemas agrarios, de su conexión con la industria y de las prácticas alimentarias pueden ayudarnos a entender las razones por las cuales la patata quedó al margen de la dieta humana en las regiones que hemos mencionado, mientras que, paradójicamente, su cultivo cubría extensiones de tierra cada vez más importantes. Como veremos, en el Bassin Parisien fue una planta industrial, ya que una considerable parte de la cosecha era utilizada para la producción fécula, la cual, a su vez, era empleada por los fabricantes de alcohol (Burton in Salaman, 1985, pp. 564-572; Peckham, 1986) ${ }^{4}$. En este sentido, este trabajo tiene entre sus objetivos identificar las lógicas que presidieron el funcionamiento de los sistemas agrarios, lo que nos permitirá, entre otras cosas, comprender en qué condiciones la patata fue susceptible de llegar a ser relevante en el régimen alimentario. Conforme a ello, en la primera parte, analizaremos la evolución de su producción en la región

A pesar del interés de su trabajo, Peckham atiende mucho más a la producción de almidón a partir del trigo que a la producción de fécula a partir de la patata (el término inglés starch se puede traducir por almidón producido a partir del trigo, o por fécula producida a partir de la patata). Por otro lado, y como nos lo indican recientes investigaciones, desde el Primer Imperio, la industria se beneficiaba de un régimen de libertad mucho más amplio de lo que supone este autor (MassardGuilbaud 2010; Le Roux 2011). 
parisina a lo largo de la primera mitad del siglo XIX. En la segunda, estudiaremos la composición del mencionado régimen alimentario de la población de Île-de-France, es decir, de los tres departamentos situados en el centro del Bassin Parisien (Seine, Seine-et-Oise y Seine-et-Marne) ${ }^{5}$, a partir de resultados — no publicados, y hasta hoy no explotados - procedentes de la Encuesta Agrícola de $1862^{6}$. Sobre esta base, trataremos de explicar por qué las patatas constituyeron un elemento tan marginal en la dieta de los parisinos, sobre todo, cuando sabemos que desde al menos mediados del siglo XIX eran consumidas con toda normalidad en el este de Francia y Bretaña. En la tercera parte, y través del ejemplo del Bassin Parisien y Lorena, mostraremos que el tubérculo era una planta industrial destinada a la producción de fécula y al engorde de los animales.

\section{La producción de patata en Île-de-France en el siglo XIX}

Como se aprecia en el gráfico 1, las superficies dedicadas a la patata en la región parisina aumentaron rápidamente en el curso de la primera mitad del siglo XIX. En 1810-1820 ocupaban todavía un lugar tan marginal en las rotaciones de cultivos que, en determinados años, las estadísticas de la época ni las mencionan. Bajo la Monarquía de Julio (1830-1848), comienzan a aparecer ya sistemáticamente en dichas rotaciones, de tal modo que en torno a 1850 ocupaban cerca del 10\% de la superficie dedicada a los cultivos alimentarios?

Aunque, como va dicho, las patatas apenas parecen citadas en las estadísticas oficiales de comienzos del siglo XIX, no cabe duda de que eran cultivadas a pequeña escala. Luego, a medida que la centuria avanzó, su cultivo se disparó. En 1816, por ejemplo, en los dos principales departamentos agrícolas de la región parisina su producción se estimaba en unos 300.000 quintales al año. Sin embargo, hacia 1850 esa producción era ya de unos dos millones de quintales, cantidad que en 1860 llegaría a situarse en torno a los tres millones al año (gráfico 2). En otras palabras, en cuarenta y cinco años la producción se multiplicó por diez.

Pese a que pueda llegar a dudarse de la fiabilidad de las estadísticas de principios del XIX, parece evidente que a mediados de siglo la patata no era un cultivo secundario, confinado en las huertas de las grandes granjas o en las pequeñas explo-

Los departamentos franceses se dividen en distritos, los cuales a su vez se dividen en cantones, y cada cantón reúne en su seno un conjunto más o menos amplio de municipios.

No confundir esta encuesta con la de 1852 .

En el gráfico no se tienen en cuenta la avena y la cebada. La avena ocupaba un lugar muy importante en las rotaciones de cultivos. En la región parisina estaba destinada exclusivamente a alimentar las caballerías tanto urbanas como rurales. 


\section{Gráfico 1. Superficie ocupada por diferentes cultivos en las rotaciones} agrícolas de Seine-et-Oise y Seine-et-Marne, en Île-de-France [1]

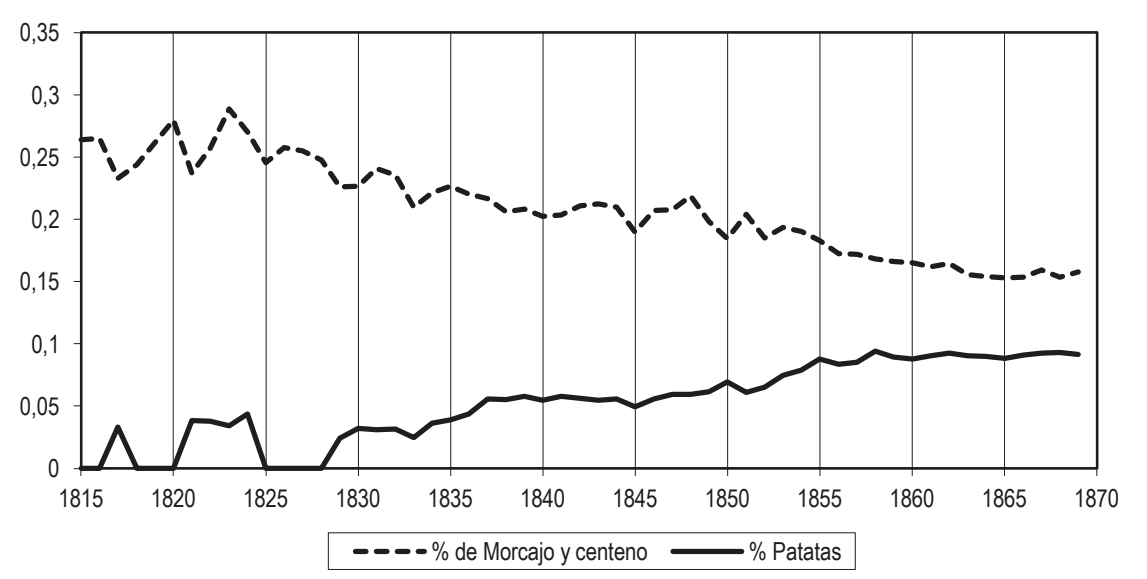

[1] El total de las superficies representan la suma de las superficies ocupadas por el trigo, el morcajo, el centeno y la patata.

Fuente: Récoltes des céréales et des pommes de terre, de 1815 à 1876, Ministère de l'Agriculture, Paris, 1887.

Gráfico 2. Evolución de la producción de patatas en la región parisina

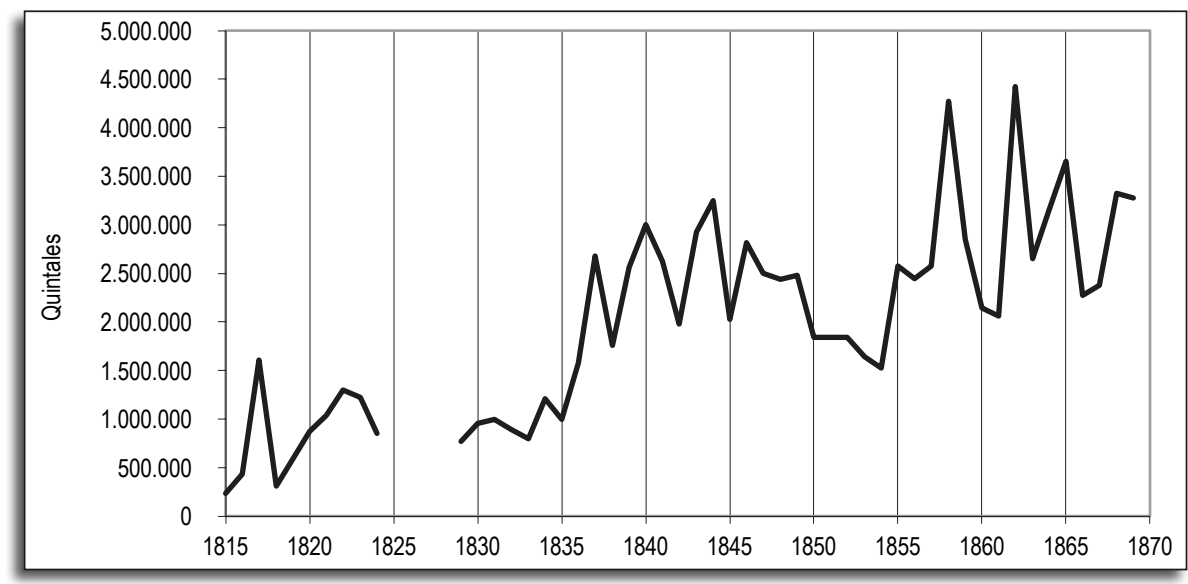

Fuente: Récoltes des céréales et des pommes de terre, de 1815 à 1876, Ministère de l'Agriculture, Paris, 1887. 
taciones agrícolas de los jornaleros. En la década de 1850, si no antes, el tubérculo se cultivaba a campo abierto y ocupaba varias decenas de miles de hectáreas. Una extensión que ahora representaba alrededor de un séptimo de la superficie agrícola dedicada al trigo, equiparándose así a la de la cebada y el centeno.

Quizás, haya quien pueda querer interpretar la evolución de la producción de la patata como un indicador del cambio producido en el régimen alimenticio de los habitantes del Bassin Parisien, visto que el incremento de esa producción coincide en el tiempo con el descenso operado en las superficies consagradas al cultivo de centeno y morcajo (una mezcla de trigo y centeno). De seguir esta idea, habría que suponer entonces que la patata habría procedido a sustituir poco a poco a los cereales menos apreciados por la población, pero mucho nos tememos que las fuentes no avalan esta interpretación.

\section{La patata en la dieta de los habitantes de Île-de-France}

A pesar del aumento en las cantidades producidas en los dos primeros tercios del siglo XIX, desde un punto de vista alimentario el consumo del tubérculo continuó siendo marginal. Los tres o cuatro millones de quintales cosechados cada año eran muy poca cosa en relación a las necesidades cotidianas de París y su región, más aun, cuando una parte importante de lo recolectado - aproximadamente un 10\% del total - tenía que ser empleado como semilla. En este sentido, mejor sería comparar esa producción con las cantidades de harina llegadas a la ciudad para su abasto. Apreciamos entonces que París consumía cada mes unos 120.000 quintales de harina, lo que en un año suponía cerca de 1,5 millón de quintales. Por su parte, el millón de habitantes que poblaban sus suburbios y los departamentos de Seine-et-Oise y Seine-etMarne, consumían cantidades de harina muy importantes, equivalentes sin duda a las de París. Podemos estimar así en casi tres millones de quintales la cantidad de harina destinada anualmente al consumo de la ciudad y su entorno, o lo que es igual, unos 180 kilógramos de pan al año por habitante, mujeres, niños y ancianos incluidos. Si atendemos a que el poder calórico de la patata es tres veces más bajo que el del pan, es obvio que esos 3-4 millones de quintales producidos cada año en la región representarían una parte ínfima de la ración alimentaria de su población.

El almacenamiento y consumo de la patata planteaba por lo demás numerosos problemas, ya que el primero tenía que protegerla del frío y del calor con el fin de asegurarse que fuese apta para el consumo y no germinase, mientras que el segundo, el uso alimentario, implicaba igualmente la resolución de problemas bastante espinosos. Por ejemplo, si se la quería cocinar, se necesitaba una fuente de energía, así como un cierto tipo de grasa comestible que la acompañase - mantequilla, aceite 
vegetal o grasa animal-, puesto que, en sí misma, la patata contiene muy pocas calorías (para el caso de Irlanda, véase Mokyr, 1981). Ahora bien, Île-de-France era una zona donde esas grasas eran caras, mucho más que en sus respectivos lugares de producción - caso de la mantequilla en Normandía y Bretaña o en las zonas de montaña; del aceite vegetal, sea de adormidera o colza, en Normandía y el norte de Francia; o del aceite de oliva en la cuenca del Mediterráneo-. La citada fuente de energía también era cara, visto, por ejemplo, que el abastecimiento de leña a París se realizaba desde bosques situados a cientos de kilómetros, mientras que el carbón se importaba del norte de Francia y Bélgica, ya fuese por vía fluvial o por tren (acerca de la evolución del precio de la energía a largo plazo, véase Allen, 2009, pp. 81-105). En estas circunstancias, no sorprenderá que el consumo de patatas fuese mucho más importante en lugares como Bretaña, Nord-Pas-de-Calais y el este de Francia, donde las grasas animales o vegetales y el combustible - leña, turba o carbón — eran mucho más abundantes y baratos. En estas zonas, los campesinos podían disponer de esas grasas y combustible, y convertir fácilmente el tubérculo en elemento más de su dieta cotidiana. Por el contrario, en la cuenca de París todo iba en contra de su consumo masivo, no sólo en la ciudad, donde muy pocas mujeres lo cocinaban, sino también en las zonas rurales que la rodeaban. Por lo demás, es probable que entre las clases populares parisinas ese consumo se limitase al que les proporcionaban los taberneros y vendedores ambulantes de patatas fritas, lo que no sucedía en las afueras de la capital, donde apenas se comían.

La Encuesta Agrícola de 1862 nos ofrece numerosas informaciones acerca de la dieta de la población rural (Figura 1). En su cuarta parte, que nunca llegó a publicarse, los administradores locales, a menudo los alcaldes de los municipios o los jueces de paz de los distintos cantones, debían indicar los elementos que formaban parte del régimen alimentario de cinco tipos de varones adultos: los «granjeros acomodados», los «pequeños propietarios cultivadores», los «aparceros», los «obreros del campo que se alimentaban en las granjas» y «los jornaleros» (sobre la Encuesta Agrícola de 1862, Herment, 2017; sobre la de 1852, Grantham, 1989). Los datos que nos aportan no son fiables, pero, globalmente, los administradores locales se afanaron por responder con la mayor precisión posible a las cuestiones planteadas, si bien, a veces, reconocieron no ser capaces de ofrecer estadísticas relevantes. Muchas veces, tal y como se aprecia en la imagen 1, las informaciones cuantitativas que aportan se acompañan de un comentario que permite precisar ciertos aspectos importantes de la dieta o aclarar determinadas costumbres culinarias. En ocasiones, ese comentario describe las prácticas alimentarias o agrarias a nivel de todo el distrito. Por nuestra parte, hemos examinado las respuestas contenidas en los cuestionarios comarcales de tres departamentos del centro del Bassin Parisien, es decir, de Île-de-France (Seine-et-Marne, 
Seine-et-Oise y Oise), tres de Bretaña (Côtes-du-Nord, Finistère y Morbihan) y tres de Lorena (Meurthe, Moselle y Vosges) ${ }^{8}$.

Como puede verse en la imagen 1, los administradores locales no tenían por qué especificar la cantidad de patatas consumidas a diario en los territorios que tenían a su cargo. Solo si lo estimaban necesario podían incluir esa información, bien fuese en la línea correspondiente a «hortalizas» o a «otros comestibles», o bien bajo la forma de comentarios que acompañaban a la estadística. Cuando se decidían por esta última opción, casi nunca indicaban cantidades, con lo que resulta imposible hacerse por esta vía con una idea aproximada de la importancia que tuvo el consumo la patata. En cambio, nada nos impide suponer que la frecuencia de las menciones relativas a ese consumo reflejaría, grosso modo, la posición que el tubérculo ocupaba en la dieta de la población.

\section{Imagen 1. Cuarta parte de la Encuesta de 1862 Comarca de Sarzeau (Morbihan)}

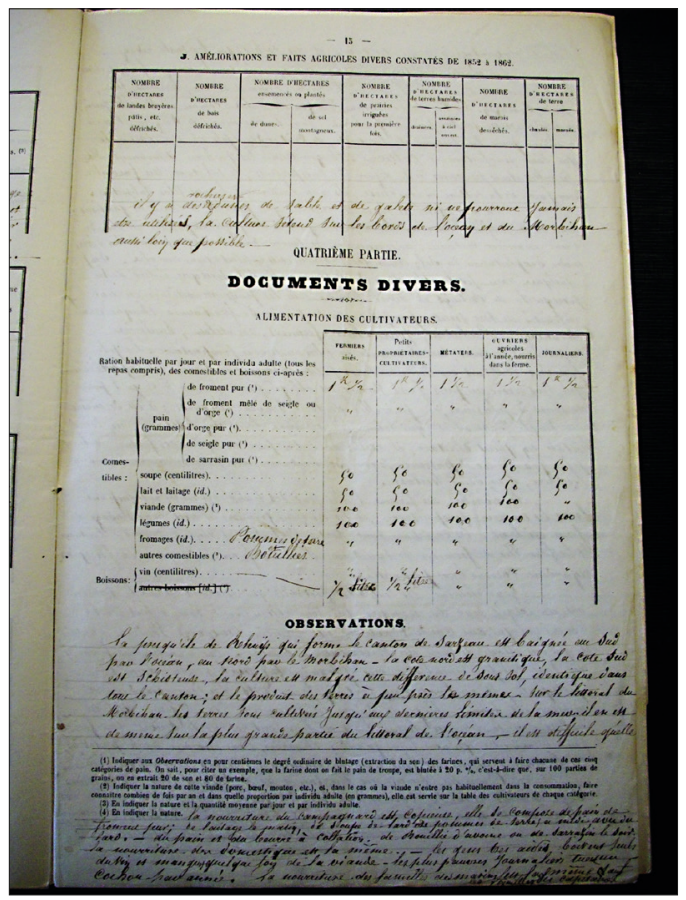

Fuente: Archives Nationales, F/11/2707.

Tras la derrota de 1870, los departamentos de Moselle y Meurthe desaparecieron. Se sustituyeron por el departamento de Meurthe-et-Moselle. A día de hoy, no hemos encontrado rastro alguno de los cuestionarios del departamento de Meurthe. 
Partiendo de esta base, procedimos a cartografiar todas las menciones referidas al mencionado consumo de patatas en los departamentos indicados.

Como puede apreciarse en el mapa 1, lo sucedido al respecto en la región parisina se opone a lo ocurrido en el oeste y en el área montañosa del departamento de los Vosgos. En Île-de-France y el sur de la Picardía, la patata sólo se nombra una vez como parte de la dieta habitual de los hombres adultos. Y a la inversa, en Bretaña y en la mencionada área montañosa de los Vosgos, se la cita con bastante frecuencia. Es más, en la zona de habla alemana del departamento de Lorena, su consumo se asocia con el de alcohol — bajo la forma de aguardiente de patata-, mientras que en los comentarios de Bretaña se la presenta como uno de los sustitutos del pan, la papilla de avena o las crepes elaboradas con trigo sarraceno.

\section{Mapa 1. Menciones a la patatas en la dieta de los varones adultos del este de Lorena, la región parisina y el oeste de Bretaña}

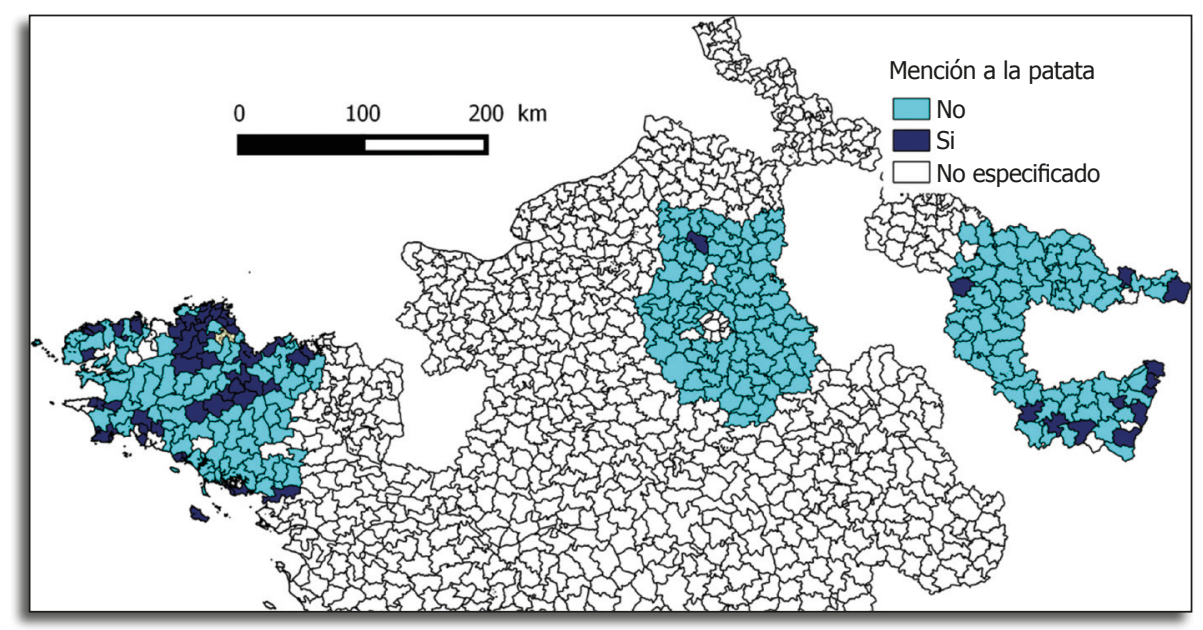

Fuente: Archives Nationales, F/11/ 2700, 2702, 2707, 2708, 2709, 2710 y 2712.

\section{Los usos de la patata: ¿planta alimenticia o planta industrial?}

Si la Encuesta Agrícola de 1862 no nos permite conocer las cantidades de patatas consumidas por los varones adultos, la primera parte de la misma, consagrada a los cultivos, se convierte en cambio en un instrumento ideal para identificar los usos que se le dieron al tubérculo en los distintos cantones considerados. Y es que los administradores locales tenían la obligación de ofrecer mucha información acerca 
de esos cometidos, en particular de los cereales y los alimentos de fécula, caso, por ejemplo, de la superficie dedicada a cada uno de ellos, su rendimiento $\mathrm{y}$, lo que nos interesa más, el uso que se les daba a sus cosechas. Al respecto, la encuesta distinguía cuatro posibles usos: semillas, cantidades consumidas por los hombres, las que lo eran por animales y, finalmente, las empleadas por la industria.

Con la intención de garantizarnos la fiabilidad de esta información, hemos procedido a comparar las cantidades empleadas como semilla y el total de la producción, el cual puede calcularse multiplicando la superficie cultivada por el rendimiento por hectárea. Ambas cantidades, semillas y total de la producción, fueron establecidas de manera independiente, por lo que si el grado de correlación entre las dos series resultantes es elevado, podremos suponer que los datos sobre el uso de la patata son fiables.

En el gráfico 3 se presenta ese grado de correlación, el cual demuestra ser muy alto. Conforme ello, comprobamos entonces que, y salvo algunas excepciones, la estadística de la Encuesta Agrícola señala que aproximadamente entre un 7\% y un 15\% de la producción total se dedicaba a semilla9 .

\section{Gráfico 3. Correlación entre las semillas y la producción de patata ${ }^{10}$}

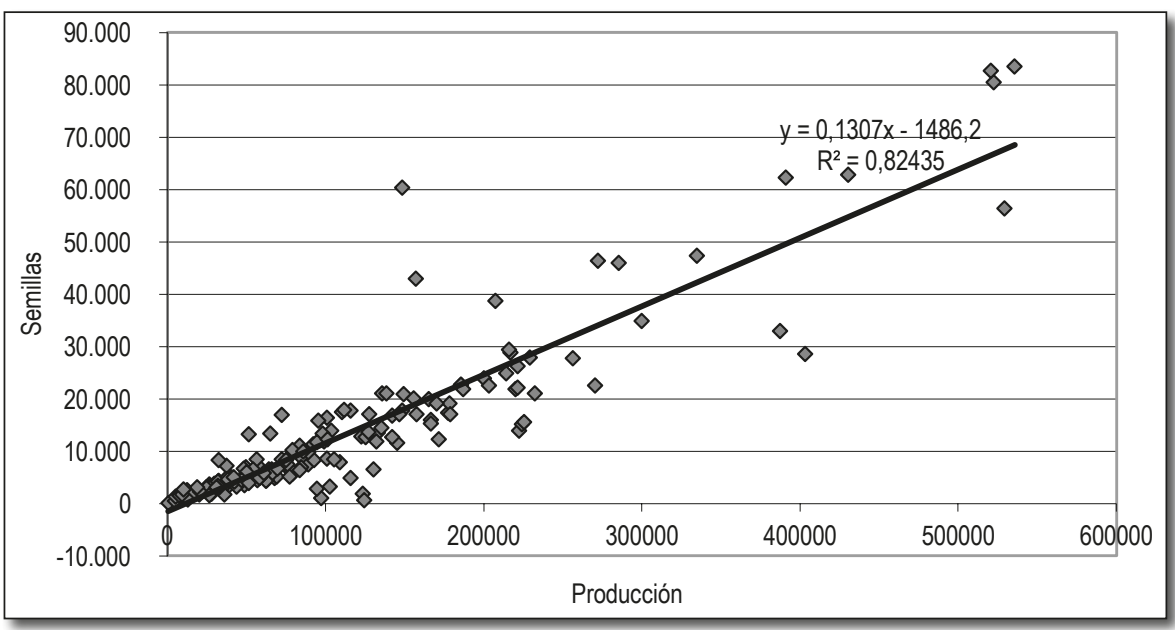

Fuente: Archives Nationales, F/11/ 2707, 2708, 2709, 2710 y 2712.

\footnotetext{
9 Por término medio las semillas representaban alrededor de un $11 \%$ de las cantidades producidas.

10 Cálculo realizado a partir de 181 cantones de siete departamentos: Seine, Seine-et-Oise, Seine-etMarne, Oise, Meuse, Moselle y Vosges.
} 
Más arriba vimos que en la región parisina la patata no era un elemento importante en la dieta de sus habitantes. Basándonos en las estadísticas de la Encuesta Agrícola de 1862 sobre el uso del tubérculo, es posible hacer un cálculo aproximado sobre la parte de la producción que se dedicaba a la alimentación. En los cantones del Bassin Parisien y del sur de Picardía, donde los usos alimentarios de la mencionada patata constituían la norma, se producía sin embargo en muy poca cantidad. La única excepción la constituía el cantón de Courbevoie, debido a la existencia en su seno de un cuartel, el cual, de por sí, determinaba un alto consumo del producto. De hecho, en la página correspondiente de la encuesta aparece anotado al margen: «cuartel».

\section{Gráfico 4. Parte correspondiente a los usos alimentarios e importancia de las cantidades consumidas en el gran Bassin Parisien ${ }^{11}$}

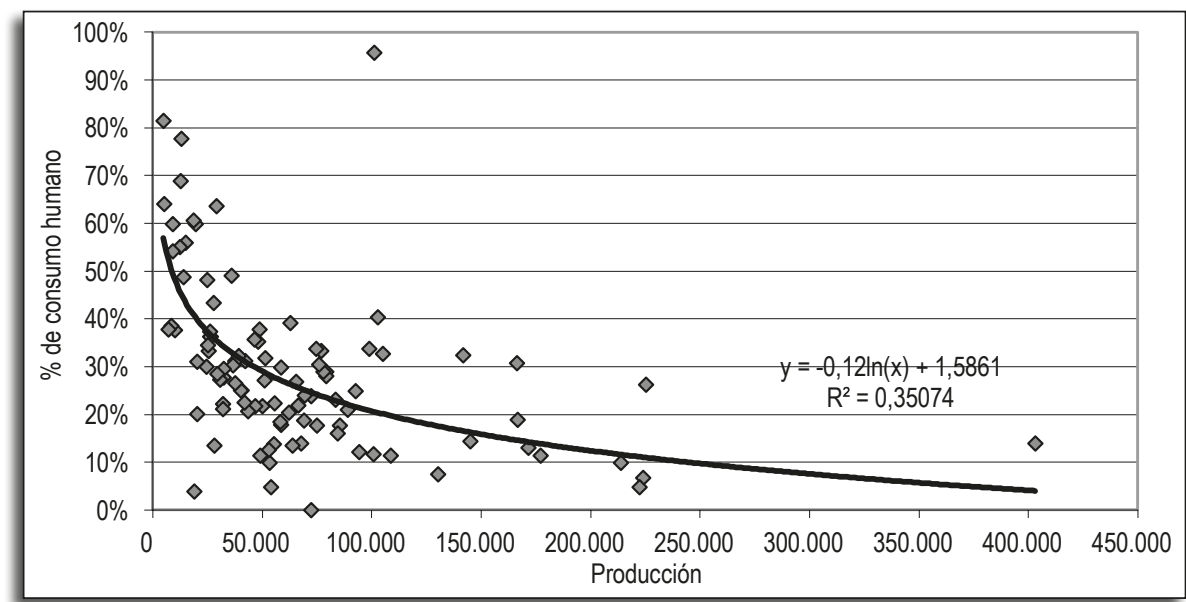

Fuente: Archives Nationales, F/11/ 2708, 2709 y 2710.

Los cálculos realizados confirman, al tiempo que permiten matizar, lo que el análisis de la dieta alimenticia revelaba: que la población del Bassin Parisien consumía patatas y que en los cantones del mismo cuya producción era elevada ese consumo también era elevado, solo que, y esto hay que tenerlo presente, eran lugares donde la parte destinada al consumo humano con respecto al total de la cosecha resultaba ser baja. Es decir, que si la patata no estaba del todo ausente de esa dieta, las superficies agrícolas de la región no se dedicaban a producir preferentemente para el consumo humano, visto que la parte destinada a éste nunca superó el 30\% del total de

$11 \quad 100$ cantones de los departamentos de Seine, Seine-et-Oise, Seine-et-Marne y Oise. 
la cosecha. La alimentación del ganado, de los cerdos en particular, es el uso que más a menudo se menciona en la Encuesta Agrícola, es más, en determinadas regiones del este de Francia ese era su principal destino. Así, por ejemplo, en los tres departamentos de Lorena mencionados, (Meuse, Moselle y Vosges), alrededor del 45\% del total de la cosecha se destinaba al engorde de los animales y solo un $21 \%$ a la alimentación de las personas ${ }^{12}$. Por el contrario, en Bretaña, lo esencial de la producción se dedicaba al consumo humano.

Ni en el Bassin Parisien ni en el departamento de los Vosgos, salvo en su parte más montañosa, la patata era un elemento esencial en la dieta de la población rural. Y aunque, en Lorena, en el cantón de Clermont, el presidente de la comisión encargada de la confección de la Encuesta de 1862 señalaba que «es una parte capital de la comida de la población», este tipo de anotaciones es muy poco común, excepto, como va dicho, en la montaña de los Vosgos. De hecho, solo en esta última y en Bretaña el tubérculo se impuso como alimento básico, en buena medida debido a que en ambos casos se daba la comentada coincidencia entre su cultivo, la existencia de combustible abundante (caso de la turba y/o la leña) y la presencia de una importante producción de grasa (leche, mantequilla y queso). Tres factores que permitieron su conversión en un producto que era consumido en abundancia ${ }^{13}$.

En los cantones donde se dedicaba una parte importante de la superficie a su cultivo y los rendimientos obtenidos eran altos, la patata se destinaba a la industria de la fécula, así sucedía en los Vosgos, en Oise y en los alrededores de París. En los cantones donde más del 50\% de la producción iba a parar a la mencionada industria, los rendimientos por hectárea se situaban en torno a los 193 quintales, cantidad que se reducía a solo 143 en aquellos en los que la cosecha tenía como fin la alimentación humana ${ }^{14}$.

En el mapa 2 puede apreciarse este tipo de especialización en el Bassin Parisien y en el distrito de Epinal, sito en el departamento de los Vosgos. En ambas zonas, la patata se cultivaba de manera intensiva al objeto de satisfacer la demanda de la industria. No en vano, los contemporáneos la relacionaban con la producción industrial de fécula. Por ejemplo, un individuo, un tal Delamarre, que deseaba abrir una de estas fábricas en Guyancourt, cerca de Versalles, señalaba en la petición remitida a las autoridades que «la agricultura de las llanuras de la Minière, Bouviers,

$12 \quad$ Cálculo efectuado sobre una muestra de 81 cantones loreneses para los cuales los datos acerca del uso de la patata están disponibles y son fiables. Archives Nationales (en adelante AN), F/11/2707 y 27012.

13 La explotación de los yacimientos de hulla de Lorena estaba todavía en sus inicios en esta época, si bien la existencia de los mismos era conocida desde hacía tiempo.

14 Estadística establecida sobre la base de los rendimientos. 
Guyancourt, Buc, Voisins-le-Bretonneux y Châteaufort, sacarían gran provecho de la instalación de este establecimiento de fécula» (Rabot 1870, p. 221). Por consiguiente, es obvio que en este ámbito el cultivo de la patata se concebía como un complemento de la industria.

\section{Mapa 2. Producción de patatas en el Bassin Parisien y Lorena}

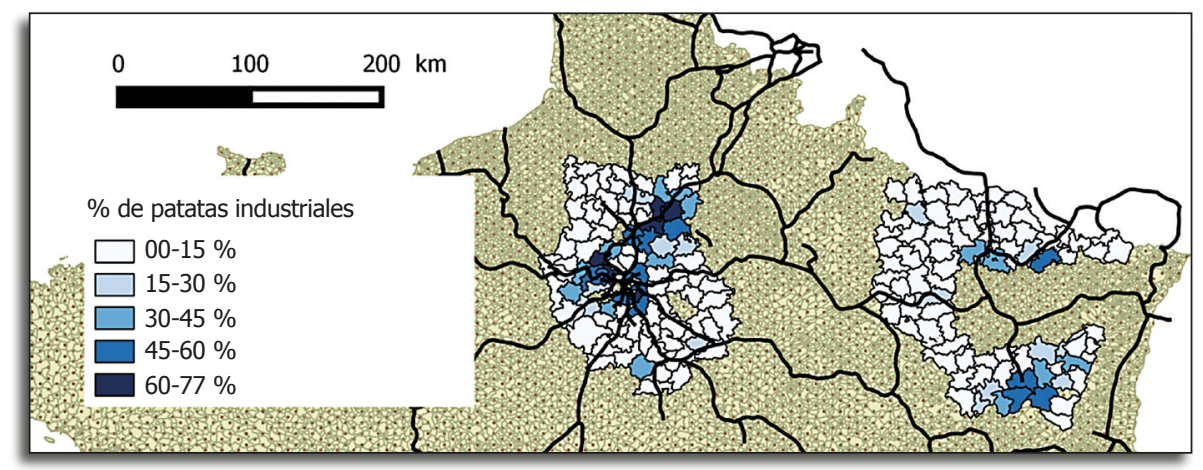

Fuente: Archives Nationales, F/11/ 2707, 2708, 2709, 2710 y 2712.

En la región de París, la geografía de la patata no obedecía prioritariamente a la necesidad de alimentar a la población de la ciudad y su ámbito de influencia. Lo cierto es que el tubérculo solo constituía una especialidad hortícola en uno o dos de sus cantones. En el centro, en Île-de-France, en los departamentos de Seine y Seine-et-Oise, la instalación de las citadas fábricas de fécula se realizaba siguiendo dos principios básicos: se disponían junto a las vías ferroviarias y a las áreas de cultivo. En dichas áreas las grandes explotaciones cerealícolas dominaban el paisaje rural, como por ejemplo en La Plaine de France, Valois, Vexin-Français (al norte de París), Longboyau y la llanura de Saclay (respectivamente al sur y al oeste de la capital) (Moriceau, 1998; Moriceau y Postel-Vinay, 1992, Herment, 2012). Algo que se puede apreciar con claridad en el mapa 3.

Las fábricas de fécula se distribuían pues en dos zonas situadas alrededor de París: al norte, en la Plaine de France, que era la parte más industrializada de los suburbios y estaba próxima a las regiones cultivo cerealícola, y al sur y suroeste, en Longboyau y la llanura de Saclay. Si la especialización de Longboyau es muy neta y clara en el map. 3, la de Saclay se nos escapa por la falta de datos sobre la producción agrícola del cantón de Versalles. No obstante, uno puede hacerse una idea acerca de la relevancia de la obtención de fécula en los alrededores de Versalles gracias al ma- 
Mapa 3. Cultivo de la patata y ubicación de las fábricas de fécula en Île-de-France

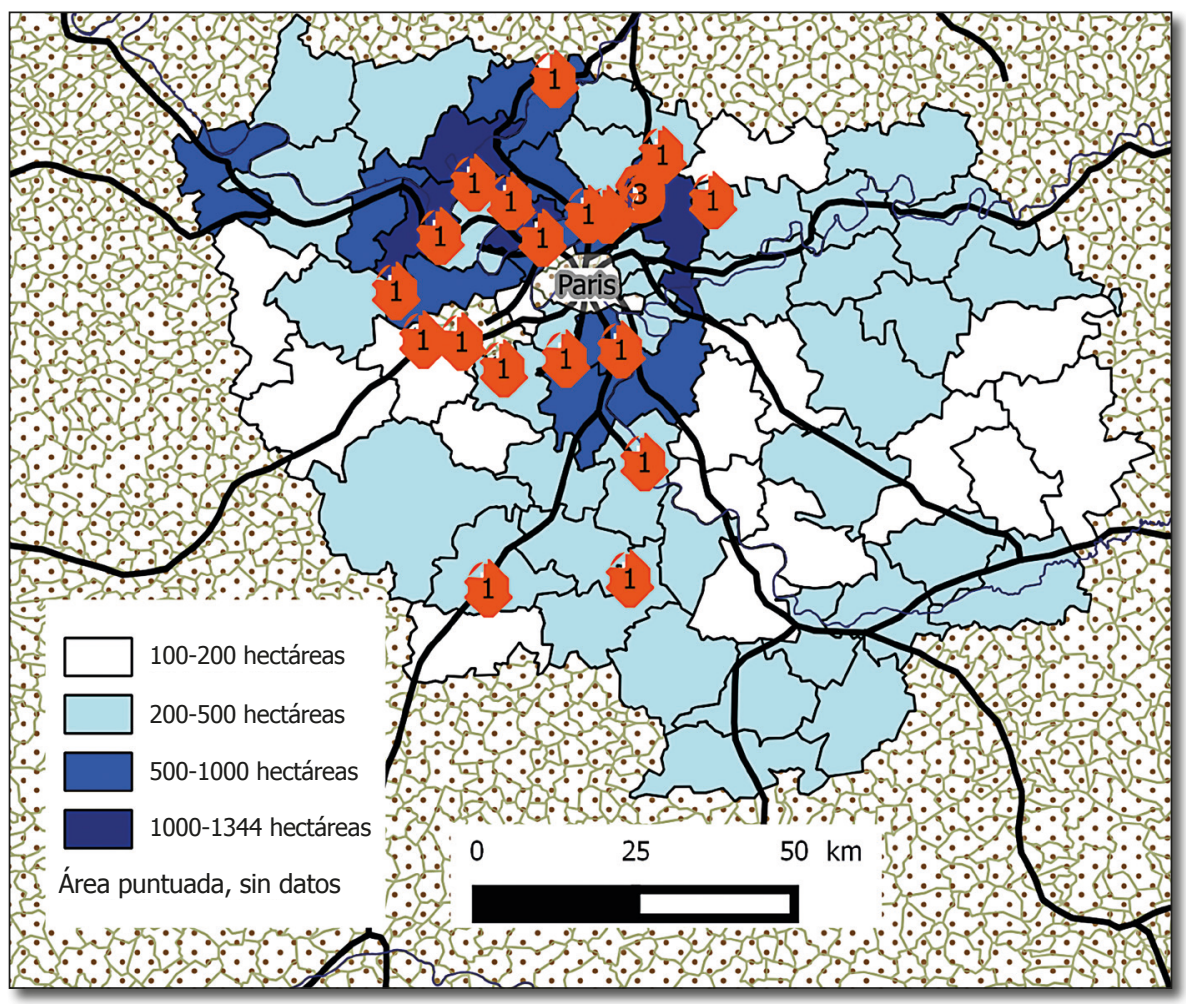

Fuente: Archives Nationales, F/11/2709 et 2710. Annuaire Firmin-Didot, 1862.

nejo de la contabilidad de la fábrica de Dailly, en Trappes ${ }^{15}$. El tal Dailly era uno de los grandes agricultores del Bassin Parisien. Desde sus granjas dirigía un servicio de transportes urbanos cuya caballería - compuesta por varios cientos de percherones y jamelgos - era alimentada por lo que éstas producían (Bouchet, 1993, pp. 111-112). Asimismo, tenía también entre sus propiedades una destilería.

En marzo de 1860, la contabilidad de la granja de Bois-d'Arcy nos indica que Dailly había plantado 7.4685 hectáreas de patatas destinadas al sostén de su fábrica de fécula. Aunque desconocemos cual era la extensión total del área cultivada en la granja de Trappes, sí sabemos que en 1859 su tamaño rondaba las 14 hectáreas ${ }^{16}$. Por

15 Archives Nationales du Monde du Travail, Lille, Ferme de Bois-d'Arcy, 19AQ/41, y Ferme de Trappes, 19AQ/46.

16 Bois-d'Arcy y Trappes se encuentran en el cantón de Versalles, a unos 10-15 kilómetros de la mencionada localidad de Versalles. 
tanto, nuestro hombre utilizaba aproximadamente unas 20 hectáreas para cultivar patatas. A razón de 190 a 200 quintales por hectárea, una productividad que era normal en la región, la cosecha oscilaría entre los 3.800 y los 4.000 quintales, el grueso de los cuales se destinaba al suministro de materia prima para la mencionada fábrica. Sea como fuere, lo cierto es que el ejemplo que nos proporcionan estas dos granjas, pone de manifiesto que el cultivo del tubérculo en la zona estuvo estrechamente ligado al surgimiento de la industria de fécula.

Si por un momento dejamos a un lado el caso parisino, apreciamos que la situación vivida en el departamento de los Vosgos es particularmente sintomática de los determinantes industriales y socioeconómicos derivados de los usos atribuidos a la patata. En las llanuras de este departamento - en los ayuntamientos de Epinal y Mirecourt-, se la cultivaba como una planta industrial. El juez de paz de Xertigny nos indica al respecto que:

El cultivo de las patatas ocupa más del doble de superficie que hace diez años. Se debe a la creación de las fábricas de fécula, que se elevan a 21 en esta comarca. La introducción de esta industria hizo que se convirtiesen en campos de cultivo los $4 / 5$ de los pastos y pastizales, los cuales entraron, de este modo, en un ciclo de rotación de cultivos ${ }^{17}$.

En esta área, el precio del tubérculo venía determinado por la importancia de la demanda de la poderosa industria allí instalada. El mismo juez de paz anterior, añade:

«La mayor parte de las féculas se entrega a las tejedurías de algodón, o, como estas últimas industrias están paradas actualmente, la fécula ha caído de precio y, consiguientemente, las patatas bajaron a 2 francos el hectólitro» ${ }^{18}$

Este ejemplo nos remite a un esquema de expansión de la patata muy similar al encontrado en el caso parisino, ya que la demanda industrial era la que determina la extensión del cultivo y la que sentaba las condiciones para su prosperidad.

En cambio, en la montaña de los Vosgos, y si hemos de creer las palabras de Joseph Ferry, alcalde de Saales, capital del cantón, situada a caballo entre el límite lingüístico del francés y el alemán, la patata era un manjar. Así, canta sus maravillas en los siguientes términos:

«La patata, considerada en todos sus aspectos, es el mayor y el más rico cultivo de estos montes.

AN F/11/2712.

AN F/11/2712. 
Por su calidad, que no tiene igual, constituye el principal alimento de casi todos los habitantes del distrito de Saint-Dié.

Se usa para todo en la alimentación del pobre, del obrero, del jornalero y del cultivador poco acomodado, en fin, es el pan de los pobres [...].

Asimismo, se consume en gran cantidad en las familias acomodadas del campo [...]. También se encuentra a menudo, si bien en menor cantidad, en la mesa de los magistrados y los altos funcionarios» ${ }^{19}$

Tal y como señaló W. G. Burton en la History and Social Influence of the Potato, y como nos lo indica el ejemplo de los Vosgos, los usos de la patata eran múltiples, y a veces complementarios, pero a menudo más o menos exclusivos y excluyentes. En determinados contextos, la producción de alcohol era uno de los más relevantes (Burton 1985, pp. 564-572), lo que fue especialmente cierto en la Europa del este (Miodunka, 2014). Por lo que sabemos, en Francia este uso llamó poco la atención de los historiadores, aunque es cierto que desde la época de la Restauración está acreditado, al punto de haber dado lugar a una importante industria tanto en París como en sus alrededores (Guillerme, 2007, pp. 141-144) ${ }^{20}$. En este sentido, por ejemplo, Anselme Payen y Alphonse Chevallier destacaron en 1826 la importancia de la producción de alcohol a partir de la fécula, a la cual consagraron varios capítulos de su Tratado de la patata ${ }^{21}$. Es más, según estos autores, el consumo del tubérculo suponía en París menos de 30 kilógramos por persona al año, destinándose los dos tercios de la patata llegada a la capital a la elaboración de alcohol (Payen et Chevallier, 1826, p. 46). De hecho, en la capital esa elaboración aumentó desde al menos 1816 (Thomas, 1822, p. 60). André Guillerme lo ha mostrado de manera muy clara: era una actividad muy importante en la época, si bien, y al contrario de lo que él afirma, sólo se prohibió a causa de dificultades temporales de abastecimiento y no por otras razones (Guillerme, 2007, p. 160)22.

19

A diferencia de lo que indicado por André Guillerme, a comienzos del siglo XIX la industria de la fécula de patata no era todavía una industria altamente capitalista, como lo prueba, por ejemplo, la pequeña empresa situada en Aubervilliers en 1817. Un agricultor llamado Demars, señaló que produjo 546,25 hectólitros de patatas con el fin de dar trabajo a sus hijos y proporcionar fécula a los vecinos. Archives Départementales de Seine-Saint-Denis, 5DM-1.

21 Sobre la importancia de la patata en la producción de alcohol, y más específicamente en la elaboración de aguardiente, véase Dubrunfaut, 1825 y 1854. Por su parte, L. F. Dufief (1829, p. 93), recalca que el uso más importante de la fécula consistía en la producción de azúcar, tónicos reconstituyentes, cerveza, vino, sidra, aguardiente y vinagre. Para ver lo sucedido en Lorena, véase Dombasle (1820). La mayor parte de los autores considera que se debe relacionar la producción de fécula y alcohol con el engorde de los animales. Así, el mencionado Dombasle (1820, p. 7) nos indica que es una de las actividades más importantes de las fábricas donde se elaboraba dicha fécula.

22 Al respecto, véase la circular enviada al prefecto el 28 de febrero de 1819, AN/F/1a68/31/6. 
No hemos encontrado datos sobre la producción de fécula o de alcohol de patata para mediados del siglo XIX. Aunque existen muchas fuentes en esas fechas que se refieren a la presencia de fábricas de fécula en la región parisina, ninguna de ellas nos permite estimar la importancia que alcanzó esa producción de alcohol. Por lo demás, las estadísticas de la industria de París publicadas en 1851 solo nos ofrecen una cifra global de la producción de fécula, junto a una breve visión cualitativa de la importancia de su uso por la industria en los siguientes términos:

«Los usos actuales del almidón y de la fécula son muy numerosos: se hace cola, empleada en grandes proporciones para el cartonaje y la encuadernación; se hace engrudo, que sirve para el apresto de las telas. Los jarabes de fécula sirven para las destilerías y, en gran medida, contribuyen a la elaboración de la cerveza [1a cursiva es nuestra]. Finalmente, la fécula de patata se emplea directamente como producto alimenticio» ${ }^{23}$

No obstante, conviene destacar que desde comienzos del siglo XIX las fábricas de fécula se fueron desplazando paulatinamente hacia las afueras de la capital (map. 3). Los archivos departamentales de Seine-Saint-Denis y de Yvelines dan buena cuenta de este movimiento excéntrico, sensible ya en la década de 1820, el cual se prolongaría durante toda la centuria ${ }^{24}$. Si en 1836 no existía ni una sola de estas fábricas en el distrito de Pontoise, a 20-50 kilómetros al norte de París, en la década de 1850 había varias, y bastante importantes, en las inmediaciones de Gonesse, a unos 15 kilómetros de la capital. En 1881, las encuestas industriales llevadas a cabo por el prefecto de la ciudad nos advierten también de su presencia en Fosses y Longuesse, localidades situadas a unos 30-35 kilómetros de París ${ }^{25}$.

Asimismo aparecen numerosas informaciones cualitativas en los periódicos de agricultura y en los comentarios sobre el estado de las cosechas o, también, en los libros publicados por los industriales al objeto de promover la producción de alcohol de patata. Por ejemplo, Augustin-Pierre Dubrunfaut recomendaba el empleo de dicho alcohol para «rectificar» el que se obtenía a partir de la uva (Dubrunfaut, 1854). Aunque en 1845 el Journal d'agriculture pratique lo desaconsejaba:

«El uso del azúcar de fécula, que la química preconizaba tanto hace algunos años, se ha topado con muchos adversarios. Se reconoció que exigía una cierta

23. Statistique de l'industrie à Paris 1851, p. 497. Véase también a Thévenin, 1863, p. 117-118. Para el uso de la fécula en la elaboración de la cerveza desde principios de los años 1830, véase a Godart, 1843 , p. 8. Asimismo, Girard, 1876, p. 18, quien denunciaba una utilización demasiado habitual en Francia de la «glucosa o azúcar de fécula» en la elaboración de la cerveza.

24 Archives Départementales de Seine-Saint-Denis, DM5/1 à DM5/11.

25 Archives Départementales des Yvelines, $15 \mathrm{M} / 8$ et 15/M/9. 
habilidad de manipulación muy difícil de encontrar; que casi siempre suponía un peligro para los vinos de calidad, a los cuales les hacía perder el bouquet $»^{26}$

En la prensa agrícola, sea en el Journal d'agriculture pratique, L'Echo agricole o Le Bulletin des halles, la patata aparece casi siempre asociada a la producción de fécula, ocupando en las preocupaciones de los periodistas y cronistas un lugar totalmente marginal la destinada al consumo humano. Los numerosos artículos que el Journal d'agriculture pratique publicó a partir de 1845 son particularmente sintomáticos de ello. Este periódico era el órgano de los propietarios de las grandes explotaciones y de los agricultores más modernos. Así, tras la aparición de la «peste de la patata», se preocupó mucho más por la calidad de la fécula, en particular de aquella destinada a la elaboración de alcohol, que por el sufrimiento y las necesidades que pudieran tener las poblaciones de Irlanda, Holanda o Flandes que padecían sus efectos ${ }^{27}$. Al respecto, y ante el avance de citada plaga, señalaba que «desde un punto de vista comercial, la posición es crítica. La fécula ya ha sufrido un incremento [de precios] considerable ${ }^{28}$.

Por el contrario, la patata destinada a la alimentación no interesaba demasiado a los periodistas y cronistas parisinos. Con todo, las cantidades que se vendieron en el mercado central de París, en les Halles, fueron aumentando, en particular durante la década de 1840. El volumen de las transacciones alcanzó en ciertos periodos de año los 15.000 quintales. Pero no hay que dejarse engañar, ya que esto apenas si equivalía a 100 gramos por persona al día, es decir, poco más de 30 kilogramos por persona al año, suponiendo que ese nivel de ventas se mantuviese relativamente estable a lo largo de todo el año. En todo caso, era una cantidad muy baja en comparación con los 180 kilógramos de pan que, por término medio, consumían anualmente los parisinos. De hecho, en los periódicos las patatas aparecían situadas entre las hortalizas o los condimentos, a veces eran nombradas al lado de la mantequilla, las cebollas, los quesos, las becadas y las alondras. Productos que no eran considerados esenciales, lo que nos indica que desde un punto de vista comercial eran consideradas un producto marginal en la alimentación humana.

\footnotetext{
$26 \quad$ Journal d'agriculture pratique, 1845, p. 143.

27 Véase, por ejemplo, Journal d'agriculture pratique, 1845, pp. 164-165, p. 186, pp. 229-239, p. 285 , etc.

$28 \quad$ Ibid., p. 132.
} 


\section{Conclusiones}

Mientras que la importancia de la patata en la alimentación animal es uno de los rasgos más característicos y conocidos de la agricultura francesa, la producción de fécula y alcohol a partir de este tubérculo ha sido casi totalmente ignorada por los historiadores que han estudiado las regiones donde predominaban las grandes explotaciones agrícolas dedicadas a la producción de cereales (Moriceau y Postel-Vinay, 1992; Herment, 2012). Su importancia en los regímenes alimentarios de las poblaciones de ciertas zonas de Europa ha ocultado sus usos alternativos, en particular en lugares como Île-de-France y Lorena, donde la elaboración de fécula fue un elemento esencial en los lazos que acabarían vinculando agricultura e industria. Lo acontecido en la región parisina es paradigmático de ello.

Si es factible pensar que a principios del siglo XIX el cultivo de la patata estaba todavía confinado a huertas y jardines, todo apunta a que durante el trascurso de su primera mitad ese cultivo procedió a invadir las grandes explotaciones del Bassin Parisien donde se plantaban cereales. Es más, en ciertas comarcas de la región llegaría a ser uno de los elementos esenciales del sistema de rotación de cultivos. Así, en 1860, ocupaba entre 20.000 y 30.000 hectáreas y su producción se elevaba a 3-4 millones de hectólitros al año. Sin embargo, pese a la importancia de las cantidades producidas, la patata ocupaba una posición muy marginal en la dieta de la población de la zona. Todas las fuentes consultadas coinciden en este punto: los parisinos y la población rural del Bassin Parisien comían pocas patatas, ya que el grueso de la producción era copada por la industria de la fécula, la cual la empleaba de múltiples maneras. En este sentido, la elaboración de alcohol parece haber sido primordial. ¿Se debería concluir de ello que los parisinos bebían lo que podríamos llamar «el vodka de Saint-Denis»? Es posible. Contrariamente a lo que afirmó en su día Jean-Louis Flandrin, la producción de alcohol de patata no era exclusiva del norte y este de Europa (Flandrin y Montanari, 1996, p. 742), visto que en esta zona se usaba a nivel industrial y no solo para «rectificar» el vino o elaborar cerveza, aunque es innegable que esta práctica era habitual.

A pesar de no ser posible investigar en profundidad todas y cada una de las implicaciones que se derivan del tema, en este trabajo hemos optado por centrarnos en uno de los aspectos menos conocidos de la agricultura de la región parisina. Alrededor de París, el cultivo de la patata, como el de la remolacha, fue industrial ${ }^{29}$. La aparición de ambos cultivos dio lugar a profundas modificaciones en los siste-

29 La remolacha cultivada en la región de París era destinada a menudo a la producción de alcohol, por el contrario, la producción de este producto en Picardía se empleaba para producir azúcar. 
mas agrarios de Île-de-France. A principios del siglo XIX, éstos se centraban en la producción sistemática de cereales - avena, trigo y, secundariamente, centeno y morcajo- - y en la ganadería ovina, tanto por la lana como por la carne. Luego, hacia 1860, el cultivo de cereales decaerá en beneficio de la remolacha y la patata. En las llanuras cercanas a París - La Plaine de France, Longboyau y la llanura de Saclay_, la patata acabará ocupando el primer lugar, ya muy lejos, estaba la remolacha, trabajada sobre todo en Valois, Multien et Brie. También en Lorena, en el departamento de los Vosgos, la patata se desarrolló como cultivo industrial en las llanuras, no así en las áreas de montaña, donde ocupaba un importante lugar en la alimentación de la población.

$\mathrm{Al}$ concentrar casi exclusivamente su atención sobre los usos alimentarios del tubérculo, los historiadores franceses han obviado capítulo importante del desarrollo y la modernización de la agricultura francesa del siglo XIX. Eso sí, aclarar que lo sucedido en Île-de-France es un caso extremo. A pesar de los esfuerzos de los agrónomos y los científicos, su población, incluidos los pobres, se negaron obstinadamente a comer patatas de manera habitual, al preferir el pan, en principio, el de trigo. Es precisamente este rechazo sobre el que conviene interrogarse. ¿Puede ser este un rasgo cultural? Si examinamos el caso parisino a la luz de lo sucedido en otras regiones francesas, cuyos habitantes consumían patatas, nos daremos cuenta de que más allá de la mentalidad, es posible que en este comportamiento haya un trasfondo económico. ¿Es una casualidad que ese consumo se haya desarrollado más en aquellas regiones que podían disponer con facilidad de grasas animales y vegetales, y de fuentes de energía baratas y asequibles? Nosotros pensamos que no. De ahí que en un próximo trabajo tratemos de establecer y de medir este aspecto con mayor precisión, gracias a la información contenida en la Encuesta de 1862. 


\section{Bibliografía}

Allen, Robert C. (2009), The British Industrial Revolution in Global Perspective, Cambridge, Cambridge University Press.

Annuaire-almanach du commerce, de l'industrie, de la magistrature et de l'administration (1862), Paris, Firmin-Didot.

BARrière, Jean-Paul (2011), « Les baraques à frites dans le Nord de la France depuis le milieu du XIX ${ }^{\mathrm{e}}$ siècle », Ferriere (de), Marc et Willot, Jean-Pierre (eds.), La pomme de terre de la Renaissance au XXI siècle, Rennes et Tour, Presses Universitaires de Rennes et Presses Universitaire de Tours, pp. 215-230.

Bennezon, Hervé (2012), La vie en Picardie au XVIII siècle : du café dans les campagnes, Paris, Les Indes Savantes.

Bergman, M. (1967), "Potato Blight in the Netherlands and its Social Consequences (1845-1847)”, International Review of Social History, vol. 12, n 3, pp. 390-431.

BOEHLER, Jean-Michel (1995), Une société rurale en milieu rhénan. La paysannerie de la plaine d'Alsace (1648-1789), tomes 1 et 2, Strasbourg, Presses Universitaires de Strasbourg.

Bouchet, Ghislaine (1993), Le cheval à Paris, Genève, Droz.

Bourguinat, Nicolas (2000), Les grains du désordre. L'État face aux violences frumentaires dans la première moitié du XIX $X^{e}$ siècle, Paris, École des Hautes Études en Sciences Sociales.

Burton, William G. (1985), in Salaman, Redcliffe, History and Social Influence of the Potato, Cambridge, Cambridge University Press, pp. 564-572.

Dubrunfaut, Augustin-Pierre (1825), Mémoire sur la saccharification des fécules. Présenté à la société royale et centrale d'agriculture de Paris, pour le concours qu'elle a ouvert sur la culture de la pomme de terre et l'emploi de ses produits, Paris, Huzard.

DUBRUNFAUT, Augustin-Pierre (1854), La vigne remplacée par la betterave, la pomme de terre, etc. pour la production de l'alcool, Paris, Guiraudet et Jouaust.

Dufief, L. F. (1829) L'art d'extraire la fécule des pommes de terre, Paris, Bachelier.

DesPlat, Christian (1992), Pau et le Béarn au XVIII siècle : deux cent mille provinciaux au siècle des lumières, tome 2, les Hommes, Biarritz, J \& D éditions.

Dubuc, André (1953), « La culture de la pomme de terre en Normandie avant et depuis Parmentier », Annales de Normandie, $3^{\mathrm{e}}$ année, $\mathrm{n}^{\circ} 1$, pp. 50-68

FAivre, Carole (2011), « La pomme de terre dans les noms de préparation culinaire en France (du XVIII siècle à nos jours) », in Ferriere (de), Marc and Willot, JeanPierre (eds.) La pomme de terre de la Renaissance au XXI siècle, Rennes et Tour, Presses Universitaires de Rennes et Presses Universitaires de Tours, pp. 115-134. 
FlANDrin, Jean-Louis et MonTANARI, Massimo (eds.), (1996), Histoire de l'alimentation, Paris, Fayard.

FRÉCHE, Georges (1974), Toulouse et la région Midi-Pyrénées au siècle des Lumières : vers 1670-1789, Paris, Cujas.

GIRARD, Aimé (1876), Exposition universelle de Vienne en 1873. Section française. Rapport sur la fabrication de la bière, Paris, Imprimerie Paul Dupont.

GODART (1843), L'art de brasser, ou Manuel donnant les moyens de fabriquer dans toutes les maisons et localités de la bière, sans avoir besoin d'appareils de brasserie, Chez l'auteur, Paris.

GrAnthAm, Georges (1989), “Agricultural Supply during the Industrial Revolution: French Evidence and European Implications”, Journal of Economic History, vol. 49, $\mathrm{n}^{\circ} 1$, pp. 43-72.

GuILlerme, André (2007), La naissance de l'industrie à Paris: entre sueurs et vapeurs, 1780-1830, Paris, Seyssel Champ Vallon.

HERment, Laurent et RonsiJn, Wouter (2015), "Seasonal patterns in food markets in north-west Europe in the second quarter of the nineteenth century: the evidence of periodic markets in France, England, and Belgium, 1820 to 1850", Agricultural History Review, ${ }^{\circ}$ 63.1, pp. 60-80.

Herment, Laurent (2012), Les fruits du partage. Petits paysans du Bassin Parisien au XIX ${ }^{e}$ siècle, Rennes, Presses Universitaires de Rennes.

HERMENT, Laurent (2017), «Vidanges et fertilisants. Le cas de la poudrette parisienne au milieu du dix-neuvième siècle ", Journal for the History of Environment and Society, vol. 2, pp. 95-126.

Kaplan, Steven (1984), Provisioning Paris: merchants and millers in the grain and flour trade during the eighteenth century, Ithaca, London, Cornell University press.

Le Roux, Thomas (2011), Le laboratoire des pollutions industrielles. Paris, 1770-1830, Paris, Albin Michel.

Massard-Guilbaud, Geneviève (2010), Histoire de la pollution industrielle en France, 1789-1914, Paris, Ecole des Hautes Etudes en Sciences Sociales.

Mathieu de Dombasle, Christophe Joseph Alexandre (1820), Instruction théorique et pratique sur la fabrication des eaux-de-vie de grains et de pommes de terre, Paris, Huzard.

Mc NeILl, William H. (1999), "How potato Changed the World's History”, Social Research, vol. 66, $\mathrm{n}^{\circ} 1$, pp. 67-83.

MeyzIE, Philippe (2007), La table du Sud-Ouest et l'émergence des cuisines régionales: 1700-1850, Rennes, Presses Universitaires de Rennes. 
MiodunKA, Piotr (2014), «L'essor de la culture de la pomme de terre au sud de la Pologne jusqu'au milieu du XIX ${ }^{\mathrm{e}}$ siècle », Histoire et Sociétés Rurales, vol. 42, $\mathrm{n}^{\circ} 2$, pp. 67-84.

MoricEAu, Jean-Marc (1998), Les fermiers d'Île-de-France XVe-XVIII siècle, Paris, Fayard.

Moriceau, Jean-Marc et Postel-Vinay, Gilles (1992), Ferme, entreprise, famille. Grande exploitations et changements agricoles XVII ${ }^{e}-X X^{e}$ siècles, Paris, Ecole des Hautes Etudes en Sciences Sociales.

Mokyr, Joël (1981), "Irish History with Potato", Irish Economic and Social History, vol. $8, \mathrm{n}^{\circ} 1$, pp. 8-29.

Mintz, Syndey (1986), Sweetness and power: the place of sugar in Modern History, London, Penguin Books.

Paping, Richard, Vanhaute, Eric et O'Grada, Cormac (eds.) (2007), When the Potato Failed. Causes and Effects of the Last European Subsistence Crisis, 1845-1850, Corn publication series, $\mathrm{n}^{\circ}$ 9, Turnhout, Brepols.

Payen, Anselme et Chevallier Alphonse (1826), Traité de la pomme de terre, sa culture, ses divers emplois dans les préparations alimentaires, les arts économiques, Paris, Thomine.

Peckham, Brian W. (1986), "Technological Change in the British and French Starch Industries, 1750-1850", Technology and Culture, vol. 27, nº 1, pp. 18-39.

RABOt, M. E. (1870), Rapport général sur les travaux du conseil central d'hygiène et de salubrité du département de Seine-et-Oise, comprenant les années 1867-68 et 69, Versailles, Ch. Dufaure.

Ronsijn, Wouter et Herment, Laurent (2016), « Les mercuriales du XIX ${ }^{\mathrm{e}}$ siècle. Le contrôle et la surveillance des prix et de l'offre de grains en France et en Belgique, 1789-1914 », Revue du Nord, n 417, tome 98, octobre/décembre 2016, pp. 812-838.

Spary, Ema (2016), Feeding France. New Sciences of Food, Cambridge, Cambridge University Press.

Statistique de l'industrie à Paris résultant de l'enquête faite par la chambre de commerce pour les années 1847-1848 (1851), Paris, Guillemin.

Tebben, Maryann (2011), «French fries et identité française : la frites et les frites en tant qu'objets littéraires et culturels », in Ferriere (de), Marc et Willot, JeanPierre (eds.) La pomme de terre de la Renaissance au XXIe siècle, Rennes et Tour, Presses Universitaires de Rennes et Presses Universitaires de Tours, pp. 231-244.

Thomas, (dit de Colmar), Charles-Xavier (1822) Observations faites à la chambre des députés, sur le projet d'expulser de Paris, les distilleries d'eaux-de-vie de pommes de terre, Paris, Imprimerie de Goetschy. 
ThÉvenin, Evariste (1863), Conseil de salubrité de la Seine. Hygiène publique, Paris, Germer Baillière.

VIVIER, Nadine (2007), "The crisis in France. A memorable crisis but not a potato crisis", in Paping, Richard, Vanhaute, Eric and O'Grada, Cormac (eds.), When the Potato Failed. Causes and Effects of the Last European Subsistence Crisis, 1845-1850, Corn publication series, n 9, Turnhout, Brepols, pp. 223-246.

Vandenbroeke, Christian (1992), « La culture de la pomme de terre en Belgique (XVII-XIX ${ }^{\mathrm{e}}$ siècle), in Flaran 12 ; Plantes et cultures nouvelles en Europe occidentale, au Moyen Age et à l'époque moderne, Centre Culturel de l'Abbaye de Flaran, Auch, pp. 115-129. 